\title{
Analysis of internal gravity waves with GPS RO density profiles
}

\author{
P. Š́cha ${ }^{1}$, U. Foelsche ${ }^{2}$, and P. Pišoft ${ }^{1}$ \\ ${ }^{1}$ Charles University in Prague, Prague, Czech Republic \\ ${ }^{2}$ Institute for Geophysics, Astrophysics, and Meteorology/Inst. of Physics (IGAM/IP) and Wegener Center for Climate and \\ Global Change (WEGC), University of Graz, Graz, Austria
}

Correspondence to: P. Šácha (sacha@mbox.troja.mff.cuni.cz)

Received: 15 April 2014 - Published in Atmos. Meas. Tech. Discuss.: 11 August 2014

Revised: 31 October 2014 - Accepted: 11 November 2014 - Published: 3 December 2014

\begin{abstract}
GPS radio occultation (RO) data have proved to be a great tool for atmospheric monitoring and studies. In the past decade, they were frequently used for analyses of the internal gravity waves in the upper troposphere and lower stratosphere region. Atmospheric density is the first quantity of state gained in the retrieval process and is not burdened by additional assumptions. However, there are no studies elaborating in detail the utilization of GPS RO density profiles for gravity wave analyses. In this paper, we introduce a method for density background separation and a methodology for internal gravity wave analysis using the density profiles. Various background choices are discussed and the correspondence between analytical forms of the density and temperature background profiles is examined. In the stratosphere, a comparison between the power spectrum of normalized density and normalized dry temperature fluctuations confirms the suitability of the density profiles' utilization. In the height range of $8-40 \mathrm{~km}$, results of the continuous wavelet transform are presented and discussed. Finally, the limits of our approach are discussed and the advantages of the density usage are listed.
\end{abstract}

\section{Introduction}

Gravity waves in geophysical fluid dynamics are usually described as a group of wave motions in a fluid where the restoring force is gravity (or so-called reduced gravity). Internal gravity waves (IGWs) as a part of them are of special importance in the atmosphere for their effects on atmospheric composition, circulation and dynamics in general.
IGWs exist in continuously stratified fluids. They can be pictured as being composed of oscillating interacting particles with mutually connected phases. The waves can propagate both horizontally and vertically under a continuous interplay between gravity and inertia and an exchange between potential and kinetic energy (Cushman-Roisin, 1994). A characteristic feature of their propagation is that the group and phase velocity are always perpendicular.

IGWs are very important for atmospheric dynamics. One of their crucial roles is that they transport angular momentum from the ground upwards. That helps to maintain the rotation of the upper parts of the atmosphere and also, in general, they contribute to restoring equilibrium and to gaining energetically more favourable conditions.

The importance of gravity waves for the dynamics of the middle atmosphere has been recognized for quite a while (Hines, 1960), and the research in this area is still very active and connected with some of the most challenging issues of earth's climate and atmospheric science. Examples are: the role of the IGWs in driving the quasi-biennial oscillation (QBO) (e.g. Marshall and Scaife, 2009), the downward coupling between atmospheric layers affecting the weather in the troposphere (e.g. Ern et al., 2011) and direct influence on the middle atmospheric climate and the acceleration of the Brewer-Dobson (BD) circulation (e.g. Garcia and Randel, 2008) that implies the necessity for incorporation of the IGWs in climate models. However, as the mesoscale waves have horizontal scales between tens of and several thousand kilometres, they cannot be fully resolved by general circulation models and have to be parameterized. Therefore, it is necessary to gather as much information about the IGWs as possible. 
A comprehensive review of the IGW's effects in the middle atmosphere up to the beginning of 21 st century is given by Fritts and Alexander (2003). In that discussion, they also state the future scientific goals of IGW research. Those are mainly observations and modelling studies of the gravity wave sources along with advanced studies of nonlinear processes influencing the wave's spectral evolution and propagation. Finally, it is necessary to incorporate all the knowledge into more accurate parameterizations.

A variety of observation techniques has already been applied in the research of wave disturbances in the atmosphere. Those include radiosonde and rocketsonde measurements, balloon soundings, radar and lidar observations and other remote sensing measurements. In the past two decades, remote sensing with occultation methods has undergone remarkable development. Signals of the Global Positioning System (GPS) are exploited by radio occultation (GPS RO) and are often utilized for studies of IGWs. In the future, the potential of these sounding techniques will most likely grow due to increasing numbers of transmitters and receiver platforms (Wickert et al., 2009).

Wu et al. (2006) categorized the GPS RO as a line of sight (LOS) sensor characterized by excellent vertical and coarse horizontal (due to LOS-smearing) resolution. Therefore, the GPS RO measurements are mostly sensitive to IGWs with a small ratio of vertical to horizontal wavelength. The observations are limited at high altitudes by ionospheric residual errors and at low altitudes by strong water vapour effects. Hence we focus on the height domain between 8 and $40 \mathrm{~km}$. Nevertheless, filtering the RO data for ionospheric correction remains a factor influencing the spectral density of the signal prior to the standard density or temperature retrieval. The highest accuracy is found in the lower stratosphere, where it is usually better than $1 \mathrm{~K}$ (Steiner and Kirchengast, 2000). The GPS RO technique provides atmospheric profiles with global coverage under all weather and geographical conditions together with self-calibration ability and long-term stability. That makes GPS RO an almost perfect tool for atmospheric monitoring (Foelsche et al., 2008).

Using GPS RO, the IGWs' description can be retrieved in a series of steps. At first, a height profile of atmospheric refractivity index is derived from bending angles. In this step local spherical symmetry is assumed and therefore a limited horizontal resolution of about $300 \mathrm{~km}$ is common to limb scanning methods (Preusse et al., 2008). Then, the refractivity index can be directly related to the density of dry air. Temperature profiles, which are usually used, are computed only subsequently from the density profiles using the hydrostatic balance and the state equation for dry air. This approach, termed "dry air retrieval", neglects the contribution of water vapour to radio refractivity, but in the height range of interest for our study this effect can be neglected (Foelsche et al., 2008).

Research on atmospheric waves using GPS RO data has expanded since the papers of Tsuda et al. (2000) and Steiner and Kirchengast (2000). According to the linear theory of
IGWs, a separation between a small wave-induced fluctuation and background field has to be performed if vertical profiles of any state quantity are used for the detection of the gravity wave parameters. The choice of the background state significantly affects the results and it is quite a complicated issue, because an artificial model could never perfectly reflect the real state of the atmosphere.

Many authors who have studied the IGWs retrieved from the GPS RO dry temperature profiles have utilized different methods for determining the background temperature. For example, Steiner and Kirchengast (2000) and many others have applied various sorts of band-pass filters with cutoffs at some specified vertical wavelengths. Gubenko et al. (2012) and Vincent et al. (1997) have used approximations by loworder polynomials for the stratospheric levels. The analysis of the tropopause region is always connected with problems because of the artificial enhancement of the wave activity (even when using the advanced band-pass filter associated with different vertical wavelengths; see Alexander et al., 2011). Schmidt et al. (2012) suggest two possible approaches to solve this problem: a separation of the profile into tropospheric and stratospheric parts and application of a filter for each region or, more appropriately, the double filtering method. The question of the background separation is an important part of our study too, and is discussed in detail in the following sections.

In this paper, we present a new method for the analysis of IGWs using density profiles. Atmospheric density is the first quantity of state gained during the retrieval of GPS RO data, and it is not burdened by additional assumptions (e.g. hydrostatic balance). Moreover, we would like to stress another advantage, which has not been discussed yet - unlike temperature profiles, the density evolution with the height is theoretically inferable by means of statistical physics. Separation of the density background not only has a physical basis but can also be computed partly analytically using our method presented in Sect. 2.

To our knowledge, this is the first study where atmospheric density profiles from GPS RO are explicitly used for the study of IGWs. Liou et al. $(2003,2005)$ and Pavelyev et al. (2006, 2009), however, followed a methodologically similar approach by studying vertical refractivity gradients based on RO data. That is unexpected since the density data should be very accurate. For example, refractivity profile intercomparisons of nearly collocated profiles with radiosondes show differences of less than $0.7 \%$ below $30 \mathrm{~km}$ altitude (Schreiner et al., 2007). Another instrument that gives direct information about density is the lidar. There are several observational studies of IGW-induced density fluctuations (e.g. Wilson et al., 1991; Sica and Russel, 1999) and theoretical papers about the determination of density fluctuation from the returned power (Gardner et al., 1989), and estimations of the response of neutral density layers to gravity wave perturbations (Gardner and Shelton, 1985; Chiu and Ching, 1978). 
The next section introduces the methodology and the data sets used to retrieve the density perturbation profiles and to analyse the IGWs. The results are described in the Sect. 3 and are discussed, with concluding remarks, in Sect. 4.

\section{Methods and data}

\subsection{Separation of the background density profiles}

In deriving the separation method we will make use of two basic facts - the general form of the density decrease with height is known from theory, and the variations of the background Brunt-Väisälä frequency squared $\left(N_{0}^{2}\right)$ are substantially small (Steiner and Kirchengast, 2000; Tsuda et al., 2000). The latter leads in various studies (e.g. Steiner and Kirchengast, 2000; Tsuda et al., 2011; Gubenko et al., 2012) to a simplification of equations where $N_{0}^{2}$ is replaced within the area of interest with one constant value. Care must be taken when the area includes more than one layer, because there is a jump of $N_{0}^{2}$ at the boundaries (in our case at the tropopause).

Let us have a background density profile $\rho_{0}(z)$ and assume that all departures from the background density are due to the response to the IGW-induced wind perturbations and are governed by the continuity equation. By assuming horizontal homogeneity and neglecting the diffusion and chemical effects, Gardner and Shelton (1985) have shown that the density response can be written as

$\rho(\boldsymbol{r}, t)=e^{-\chi} \cdot \rho_{0}(z-\zeta)$.

Here $\rho(\boldsymbol{r}, t)$ is the perturbed density at position $\boldsymbol{r}$ and time $t$ and $\chi, \zeta$ are solutions of partial differential equations (for details, see Gardner et al., 1989) that are related to the wind divergence and to the vertical displacement, respectively.

Unlike continuous lidar measurements, the GPS RO data provide a snapshot of the perturbed vertical density profile $\rho(z)$. Additionally, we will make an assumption that the background density profile could be expressed analytically in the form

$\rho_{0}(z)=\hat{\rho}_{0} \exp [-a(z) z]$,

where $\hat{\rho}_{0}$ is the background density generally at the lower boundary of our vertical profile, $z$ is the vertical distance from the lower boundary and $a(z)$ is a coefficient of the background density exponential decay. In the real atmosphere, the coefficient $a(z)$ would be primarily influenced by the ambient temperature.

In general, the buoyancy frequency at any vertical level is

$N^{2}=-\frac{g}{\bar{\rho}} \frac{\mathrm{d} \bar{\rho}}{\mathrm{d} z}$.

For our case, we will define our own slightly altered expression of the background Brunt-Väisälä frequency squared

$N_{0}^{2}(z)=-\frac{g}{\rho_{0}(z)} \frac{\mathrm{d}\left(\rho_{0}(z)\right)}{\mathrm{d} z}$.
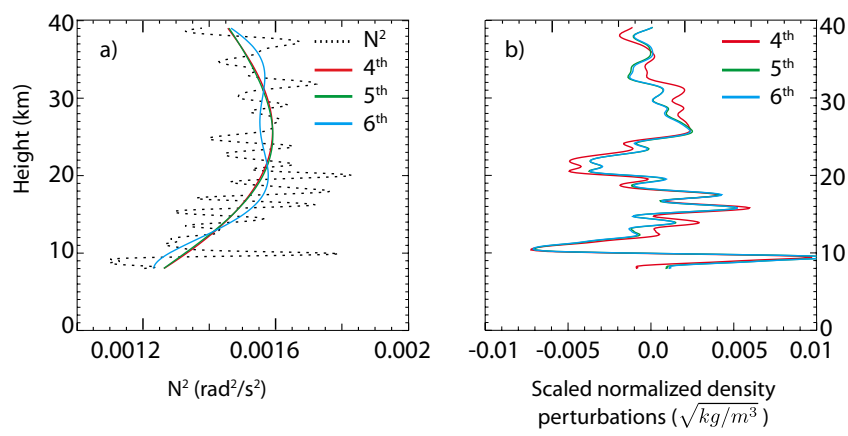

Figure 1. (a) Various (polynomial) fits of the local buoyancy frequency squared $\left(N^{2}(z)\right)$, (b) corresponding scaled normalized density perturbations.

By substituting the background density in the form defined by Eq. (2) and by omitting the notation of functional dependencies and introducing the notation $\frac{\mathrm{d} a(z)}{\mathrm{d} z}=a^{\prime}$, we gain

$N_{0}^{2}=-g\left(-a-z a^{\prime}\right)$.

Now it comes to the previously mentioned fact that $N_{0}^{2}$ generally evolves very weakly inside layers and with moderate jumps on boundaries. Hence, the frequency should be suitable for approximation by selected analytic functions. First, we must create a vertical profile of the perturbed local buoyancy frequency squared according to

$N(z)^{2}=-\frac{g}{\rho(z)} \frac{\mathrm{d}(\rho(z))}{\mathrm{d} z}$,

which is illustrated in Fig. 1. Then, by fitting the perturbed frequency, we have the formula for $N_{0}^{2}$ and we can substitute it back to Eq. (5).

Further steps will be illustrated by the simple example of fitting the perturbed local buoyancy frequency squared by a fourth-order polynomial (see Fig. 1) in the form

$A_{4} z^{4}+A_{3} z^{3}+A_{2} z^{2}+A_{1} z+A_{0}$.

After that we can substitute into Eq. (5) to get the firstorder differential equation, which can be (after making a substitution $y=a \cdot z$ ) directly solved to gain an expression for $a(z)$. Then going back to Eq. (2) we have:

$$
\begin{aligned}
& \rho_{0}(z)= \\
& \hat{\rho}_{0} \exp \left(-\frac{\frac{A_{4} z^{5}}{5}+\frac{A_{3} z^{4}}{4}+\frac{A_{2} z^{3}}{3}+\frac{A_{1} z^{2}}{2}+A_{0} z}{g}-C\right),
\end{aligned}
$$

where $A_{i}$ and $C$ are constants.

Consequently, we fit the perturbed profile $\rho(z)$ with the previously derived function of the background density's supposed dependence on the height, using the specified curve fitting procedure MPFIT introduced by Markwardt (2009). For a better and faster convergence of iterations, the numerical values of $A_{4}, A_{3}, A_{2}, A_{1}, A_{0}$ can be used as the first guess 


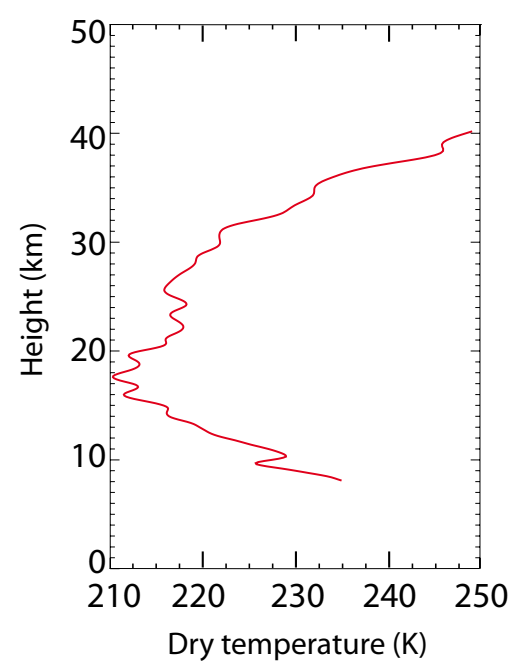

Figure 2. The dry temperature profile corresponding to the occultation event 11 March 2011, 04:08:29 UTC.

from the previously done fit of the perturbed local buoyancy frequency squared. For $\hat{\rho}_{0}$, the density value at the lower boundary can be chosen and the integration constant $C$ as a correction to $\rho_{0}$ can be assumed to be zero as a first guess.

\subsection{Data description and methodology}

The method of the density profile separation and identification of the IGWs is illustrated using data from the Constellation Observing System for Meteorology, Ionosphere and Climate (FORMOSAT3/COSMIC, Anthes et al., 2008). We have analysed a sample of 60 observational events spatiotemporally nearest (prior as well as subsequent) to the 2011 Tohoku earthquake. From this sample, one representative RO profile was chosen from 11 March 2011, 04:08:29 UTC. This occultation event was used for illustration of the results where only a single profile is used. The dry temperature profile of this event is depicted in Fig. 2; note the inversion layer around $10 \mathrm{~km}$ altitude and the significantly perturbed tropopause. The whole sample of 60 events was averaged for the analysis and results described in Sect. 3.2.1.

Results are computed using only the "dry" profiles of density and temperature. The vertical extent of our analysis is $8-40 \mathrm{~km}$ and it includes the areas of two basic inversion algorithms for GPS RO - geometrical optics (GO) and radio holography (RH). At the COSMIC Data Analysis and Archive Center (CDAAC) RH (full spectrum inversion method in this case) is applied from ground to the upper troposphere and GO from there to the top of the profile. The vertical resolution is therefore variable across the height profile, but not less than $1.5 \mathrm{~km}$ (Melbourne, 2005). For details about the algorithms and discussion of their usage possibilities see e.g. Tsuda et al. (2011).
The used methodology is limited because the tropopause is included in the investigated vertical range too. In the tropopause region assumptions of the Wentzel, Kramers and Brillouin (WKB or ray tracing) theory, that background velocity and Brunt-Väisälä frequency vary slowly over a wave cycle (Fritts and Alexander, 2003), are violated. Using this theory we are treating the wave packets as particles moving along rays (Sutherland, 2010) and using the WKB approximations we can relate the wave frequency to a wave's spatial characteristics and background atmosphere properties through a dispersion relation as shown by Fritts and Alexander (2003). Polarization relations used for the determination of the IGWs' parameters from temperature or density profile as proposed by Gubenko et al. (2011) are also a product of WKB approximations.

In the region where the ray theory is not valid, we cannot consider the amplitude envelope as slowly evolving and the relative phase, amplitude and spatial extent of the wave packet may change significantly as it evolves over time. Therefore the results are separated into the parts including the whole profile $(8-40 \mathrm{~km})$ and only the stratosphere (tropopause- $40 \mathrm{~km}$ ). In the whole vertical range, the typical analysis method of vertical wave number spectral density (e.g. Steiner and Kirchengast, 2000; Tsuda and Hocke, 2002; Tsuda et al., 2011) would not make much sense as a consequence of the facts discussed above.

Relying only on the linear theory, we will present the results of our method for background separation in the extensive region, using the continuous wavelet transform (CWT) and its skeleton as used by Chane-Ming et al. (2000). The wavelet transform was computed using the Morlet wavelet and algorithms proposed by Torrence and Compo (1998).

After the subtraction of the background density, the resulting density perturbations are further normalized and scaled by the square root of the background density to conserve the kinetic energy as suggested by Hines (1960) and discussed by Sica and Russell (1999). Nevertheless, for the results in the stratospheric region alone, we use only the normalized density perturbations. Thus, we are able to compare directly the vertical wave-number spectra of the density and temperature perturbation and evaluate them with theoretical model spectra. This distinction, where needed, is emphasized in the following text.

\section{Results}

The goal of our paper is to present our method for the background separation from GPS RO density profiles. However, the issue of background determination is not satisfactorily solvable and it is also not possible to identify with certainty the ideal background profile. Therefore, where possible, the results are shown for more types of the Brunt-Väisälä frequency background profile fits and then their qualities are discussed. 


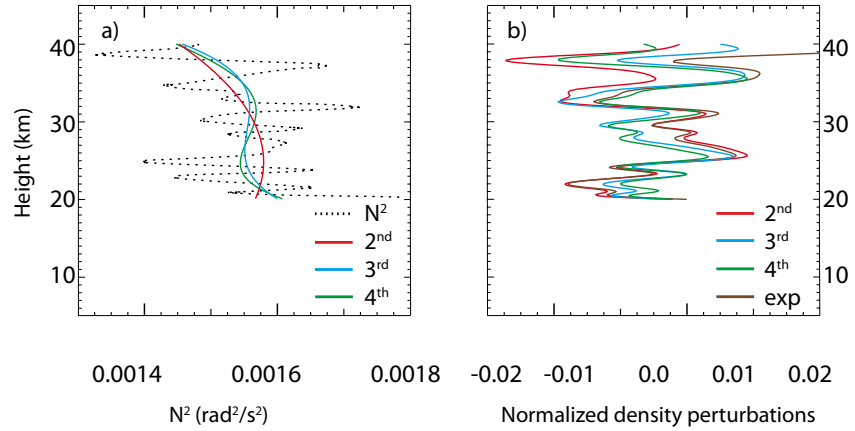

Figure 3. (a) Various (polynomial) fits of the local buoyancy frequency squared $\left(N^{2}(z)\right)$ in the stratosphere, (b) corresponding normalized density perturbations.

\subsection{The background issue}

In this subsection, the fits of the Brunt-Väisälä frequency squared are shown and discussed. Consequently, after application of our method, the resulting normalized and scaled density perturbation profiles are given. Additionally, the corresponding forms of the temperature background are computed using the equation of state for dry air and the hydrostatic balance. Conversely, background density profiles are calculated for various forms of background temperature functional dependence on altitude.

In the stratospheric region (in our case from tropopause up to $40 \mathrm{~km}$ ), the results are derived using polynomial fits of $N^{2}(z)$, starting with the second-order polynomial and ending with the fourth order. The second order is the lowest appropriate one to capture the supposed nonlinear decay of $N_{0}^{2}$. On the other hand, higher order than the fourth-order polynomial can oscillate and even the fourth-order polynomial may gain a wave-like form in this narrow region. Considering the slow changes of $N_{0}^{2}$ in the stratosphere and the limited vertical extent of our region, we must be careful not to exaggerate the polynomial order, which could result in filtering of waves with vertical wavelengths in the interval of the IGWs' likely appearance.

Chane-Ming et al. (2000) introduced a review of studies focused on the vertical wavelengths of dominant modes. In the area of our vertical range, the dominant vertical wavelengths were smaller than $10 \mathrm{~km}$. Considering the limited height range, Wang and Alexander (2010) suggested that the analysis be limited to vertical scales up to $15 \mathrm{~km}$. Steiner and Kirchengast (2000) and most other relevant studies applied the analysis threshold of the IGWs around $10 \mathrm{~km}$ of the vertical wavelength. The lower boundary of analysed IGWs' vertical wavelengths should be determined by the Nyquist frequency arising from the vertical resolution, but since the discussion about the analysis range for studying IGWs is still open (Luna et al., 2013) we will examine a broad range of vertical scales.
Having the $N_{0}^{2}$ profile in the form of a polynomial, the analytical form of the density background can be easily inferred from Eq. (8). An illustration of fits to the perturbed local buoyancy frequency squared and corresponding density perturbations are shown in Figs. 1 and 3. In Fig. 3 we added also the density perturbations resulting from the subtraction of the background in the form

$\rho(z)=\rho_{0} \exp (-a z)$

corresponding to an isothermal atmosphere. The other forms of the density background that we use are not so easily transferable in the temperature space.

For example, even when we consider the density background derived from linear $N_{0}^{2}$ profile

$\rho(z)=\rho_{0} \exp \left(-\frac{\frac{A_{1} z^{2}}{2}+A_{0} z}{g}-C\right)$.

The background temperature profile can be derived as follows. Assuming that the background state is the same for all quantities of state and that the background state is in hydrostatic balance, we use the equation of state for dry air and the hydrostatic balance equation. After solving the differential equations, the resulting background temperature profile has a quite complicated form:

$$
\begin{aligned}
T_{0}(z) & =\exp \left[\frac{\left(A_{1} z+A_{0}\right)^{2}}{2 A_{1} g}\right] \\
& \cdot K-\frac{\exp \left[\frac{\left(A_{1} z+A_{0}\right)^{2}}{2 A_{1} g}\right] g^{\frac{3}{2}} \sqrt{\frac{\pi}{2}} \operatorname{Erf}\left(\frac{A_{1} z+A_{0}}{\sqrt{2 A_{1} g}}\right)}{\sqrt{A_{1}} R},
\end{aligned}
$$

where $K$ is an integration constant, $g$ is the gravitational acceleration and $R$ is the gas constant.

If we analyse an inverse problem and try to find out which background density profile corresponds to frequently used temperature backgrounds, we discover that the polynomial fits of temperature are leading to an unphysical background in density space, generally looking like:

$\rho_{0}(z) \approx \frac{\exp [-f(z)]}{\operatorname{pol}}$

where $f$ is non-specific function of $z$ and pol denotes the original polynomial. Otherwise, if the temperature background is written using goniometric functions, the resulting density profile behaves like an exponential, but $f(z)$ has a very complicated form.

In the region from 8 to $40 \mathrm{~km}$, the task of the background separation is more complicated because we have to deal with the jump of $N_{0}^{2}$ across the tropopause. For demonstration of our method, we have chosen to fit the $N^{2}(z)$ profile with polynomials of fourth, fifth and sixth order. The corresponding density background forms can be easily inferred from Eq. (8). The local buoyancy frequency squared fits and corresponding density perturbations are illustrated in Fig. 1. 


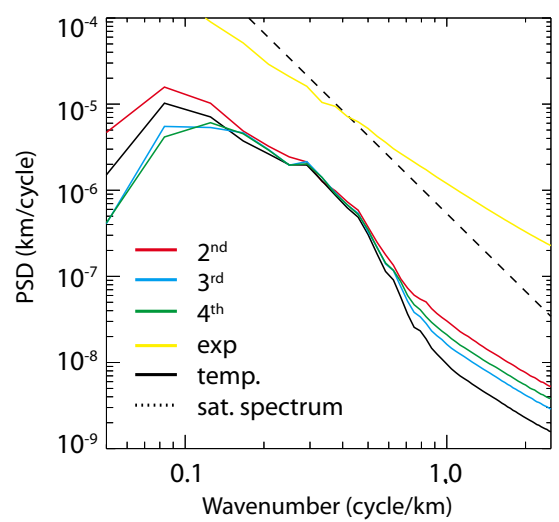

Figure 4. Vertical wave-number power spectral density for the normalized density perturbations from different backgrounds and temperature normalized perturbations compared with the theoretical saturated spectra.

As we can see in Fig. 1a, the sixth-order polynomial gains a wavelike pattern with vertical wavelength of approximately $20 \mathrm{~km}$, which is out of the interval of vertical scales normally considered to be gravity wave induced. Taking account of the temperature profile shown in Fig. 2, we can see in Fig. 1b that the tropopause (around $18 \mathrm{~km}$ ) does not cause enhancement of the gravity wave activity unlike the inversion layer around $10 \mathrm{~km}$ altitude. The method is not able to assign correctly such meteorological phenomena with sharp changes of characteristics (as inversion layers) to the background, making them an artificial source of IGWs. In general, the perturbations are not biased and are centred around zero and due to the scaling, the decrease of the wave activity in the stratosphere is clearly visible.

\subsection{Comparison with temperature}

\subsubsection{In the stratosphere}

In this section, we present a comparison between the normalized density perturbations resulting from the polynomial fits of $N^{2}(z)$ and the fit of the temperature profile with a cubic polynomial as used, for example, by Gubenko et al. (2008). We have computed the mean vertical wave-number power spectrum for the sample of 60 profiles. The vertical wavenumber spectra of the normalized density and temperature perturbations are further compared with the theoretical shape of saturated spectra for density perturbations induced by the IGWs as derived by Senft and Gardner (1991).

The mean power spectral densities (PSDs) computed for various backgrounds and dry temperature for 60 profiles are depicted in Fig. 4. The presented range of vertical wavelengths is from $20 \mathrm{~km}$ to $400 \mathrm{~m}$. The lower frequency limit was chosen due to the vertical extent of the examined area and the higher frequency limit was chosen as 2 times the Nyquist frequency of the interpolation of data to the height profile. According to Luna et al. (2013), the adequate choice of the wavelength cutoff for studying gravity waves through RO measurements is still open. For example, Wang and Alexander (2010) limited their analysis to vertical scales between 4 and $15 \mathrm{~km}$, Tsuda and Hocke (2002) applied no minimum wavelength limit but Marquardt and Healy (2005) argued that in the altitude region below $30 \mathrm{~km}$ only temperature fluctuations with vertical wavelengths more than $2 \mathrm{~km}$ can be safely interpreted as originating from small-scale atmospheric waves.

In Fig. 4 in the lower wave-number area, the influence of the background separation is dominant, with higher order fits giving lower powers. The fourth-order fit has the maximum shifted from roughly 12.5 to $7.5 \mathrm{~km}$, unlike the other density and temperature fits. That is a clear consequence of the higher possibility for this order fit to gain a wavelike form in this area (see Fig. 3a) and therefore the decrease of power in the long-wave part. For determination of whether such a long-wave mode is caused by IGW, more information (temperature, velocity, and so on) would be needed and then the polarization relations would have to be examined.

Between the vertical wavelengths of about 10 and $2.5 \mathrm{~km}$, the spectra of density from all three fits and the temperature spectrum are similar and their slopes are in good agreement with the theoretically predicted slopes. An important feature emerges at approximately $2.5 \mathrm{~km}$, where the temperature fluctuation spectrum begins to decrease more rapidly than the density spectra regardless of the fit order. This should clearly be the consequence of the usage of hydrostatic balance in the temperature data retrieval, which excludes nonhydrostatic waves and, according to Steiner and Kirchengast (2000), acts to suppress wave amplitudes. The exponential fit (isothermal atmosphere) is failing to give correct orders of PSD values as well as to capture the feature of a saturated theoretical spectrum.

\subsubsection{Over the whole profile}

Since we cannot rely on the theory (derived using WKB approximations) in the full vertical extent, we have chosen the CWT (Torrence and Compo, 1998) method for analysis of the IGWs. Using CWT, we can study the behaviour of our method for the background separation in the tropopause region and track the gravity wave activity behaviour depending on altitude. Further, for the determination of dominant modes and for studying their development with height, we have applied a method of reducing CWT to its skeleton. We have used the same setting for drawing the spectral lines as Chane-Ming et al. (2000).

In Fig. 5a-c, the CWTs are shown for the scaled normalized density perturbations, resulting from different background fits. For comparison, CWTs of the unscaled normalized density perturbations resulting from sixth- and fifthorder polynomial fit are depicted in Fig. 5d and e. Finally, the normalized temperature perturbations resulting from the 

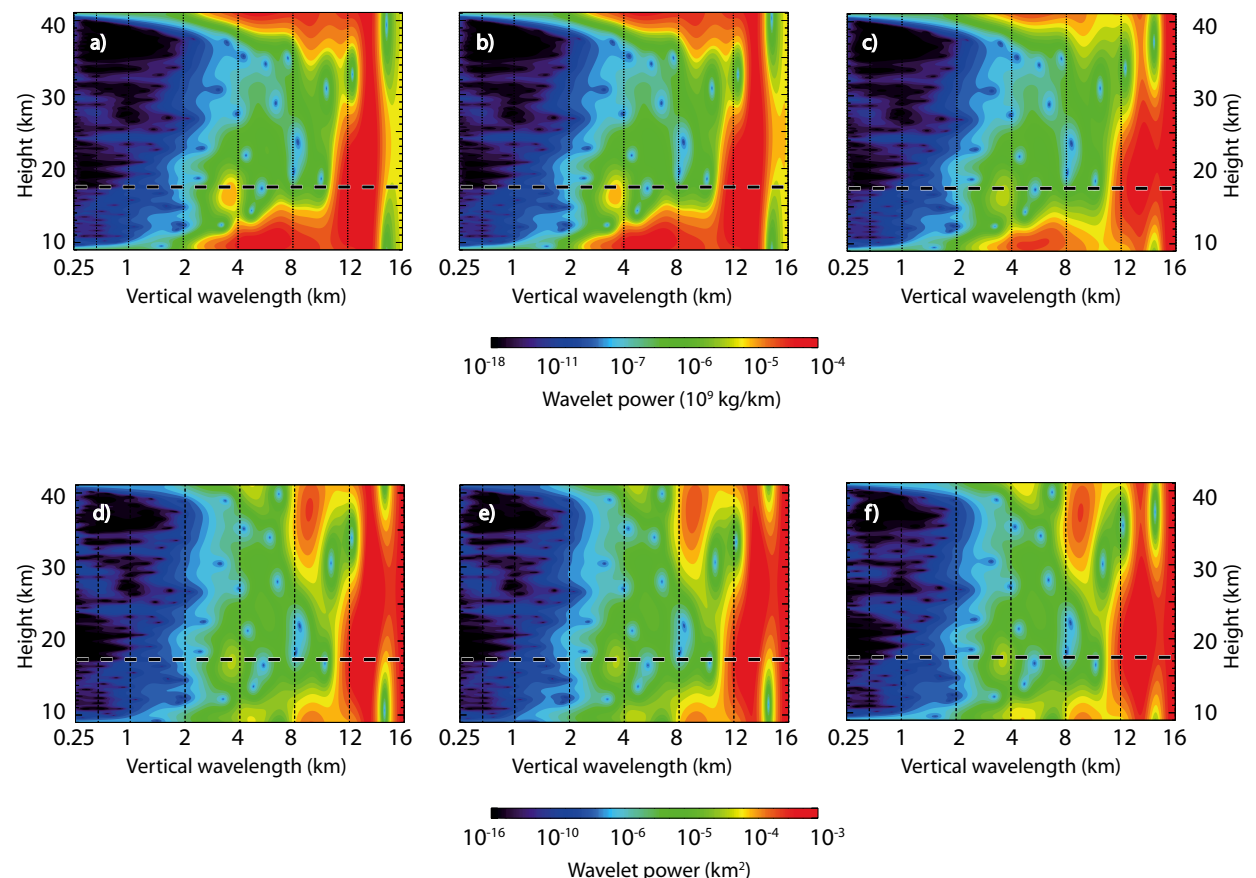

Wavelet power $\left(\mathrm{km}^{2}\right)$

Figure 5. Wavelet power spectra of various perturbations resulting from different background states: scaled normalized density perturbations resulting from the (a) sixth-, (b) fifth- and (c) fourth-order polynomial fits; normalized density perturbations resulting from the (d) sixth- and (e) fifth-order polynomial fits; normalized dry temperature perturbations resulting from the (f) sixth-order polynomial fit.

separation of the background in the form of a sixth-order polynomial are shown in Fig. $5 \mathrm{f}$.

The differences between CWTs of different fits as well as between the scaled and unscaled perturbations point to characteristic features. The differences between different fits are, as expected, most pronounced in the region of the longer vertical wavelengths. This is even more evident when comparing the skeletons of CWT in Fig. 6. In general, the difference between the scaled and unscaled perturbations is mainly connected with the theoretically predicted decrease of wave activity in the stratosphere and the shift of wave activity to longer wavelengths with height due to gradual filtering of small-wavelength IGWs above the troposphere (Fritts and Alexander, 2003). That is captured better by the CWT of the scaled perturbations. Nevertheless, there are also unexpected differences between the scaled and unscaled CWTs in the region of wavelengths around $16 \mathrm{~km}$, where the amplitude is around one order higher and constant with height for the scaled case. The temperature CWT behaves similarly to that of the unscaled density perturbations.

Spectral lines of the CWTs' maxima are plotted in Fig. 6a$\mathrm{f}$ in the same order as in Fig. 5a-f. From comparison of Fig. $6 a-c$, we can infer that the dominant mode with the wavelength around $16 \mathrm{~km}$ is probably caused by low-quality separation of background. We can argue this because in the cases of the temperature perturbations (Figs. $5 f$ and $6 \mathrm{f}$ ) and fourth-order $N^{2}(z)$ fit (Figs. 5c and 6c) where this mode is the strongest, it has height-constant wavelength regardless of the variable local stability.

Other dominant modes occurring across the different fits and across the whole profile have scales around $13 \mathrm{~km}$ and approximately from 5 to $7 \mathrm{~km}$. The skeletons should be compared with the $N_{0}^{2}$ profiles in Fig. 1a because according to, for example, Chane-Ming et al. (2000) the change of the vertical wavelength of individual modes with height should be inversely proportional to the local stability.

In our case, in the upper stratosphere, the decreasing wavelength of modes with height suggests the role of nonlinear wave processes acting to limit the amplitude which is reflected by the cessation of the wavelength growth. On the other hand, in the upper troposphere/lower stratosphere region where, in our case, the stability is growing, we can find increasing wavelength with height, which is evident for example for the mode with scale of 5-7 km in Fig. 6a. This suggests the possible enhancement of this mode's amplitude by means of wave-wave interactions or possible amplification resulting from some instabilities in the tropopause region.

\section{Summary and conclusions}

The aim of this study was to present and evaluate a method for the analysis of IGWs from GPS RO density profiles. After a description of the method for the density background separation, the methodology suitable for evaluation 

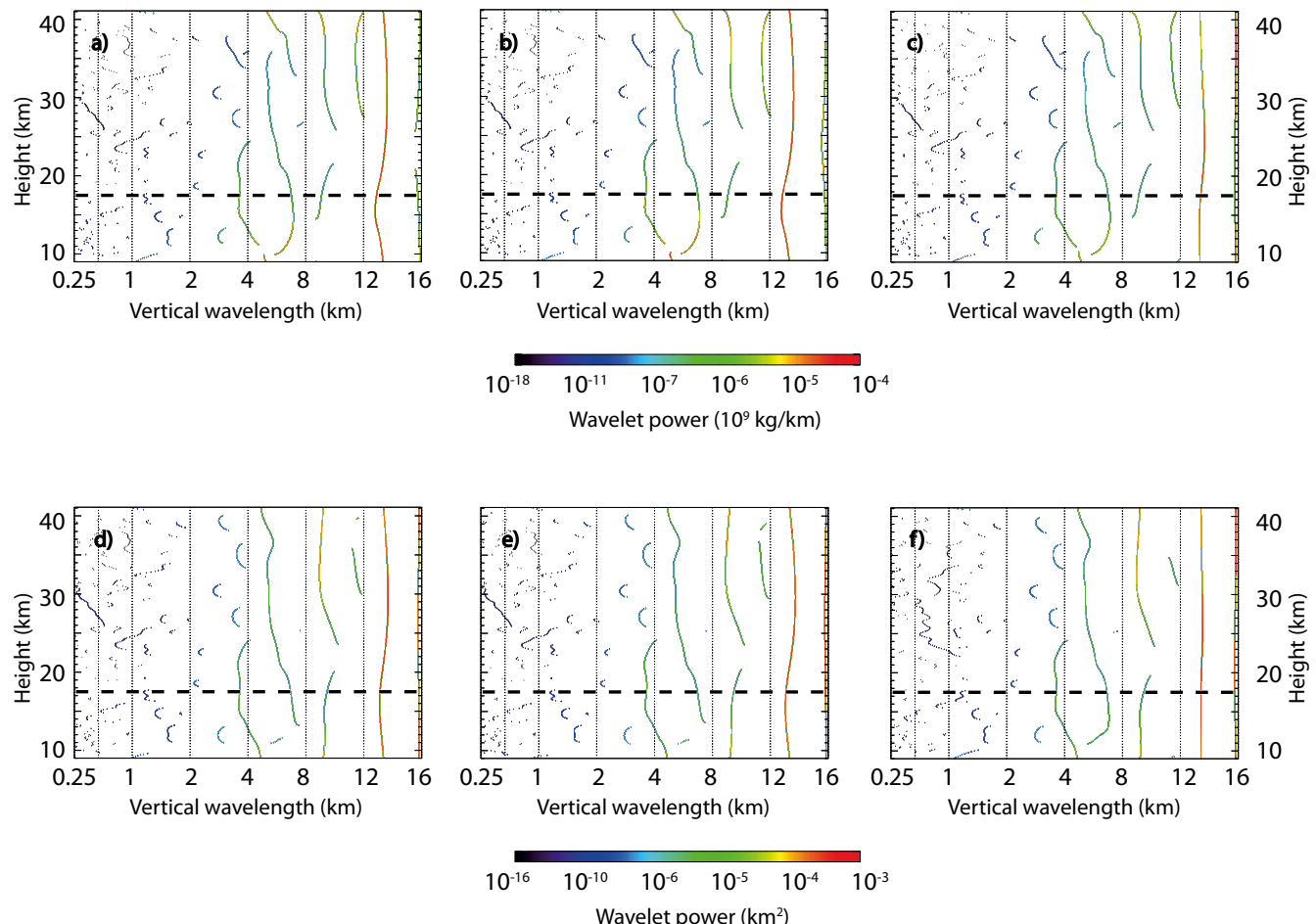

Wavelet power $\left(\mathrm{km}^{2}\right)$

Figure 6. Wavelet power spectra skeletons of various perturbations resulting from different background states: scaled normalized density perturbations resulting from the sixth- (a), fifth- (b) and (c) fourth-order polynomial fits; normalized density perturbations resulting from the sixth- (d) and (e) fifth-order polynomial fits; normalized dry temperature perturbations resulting from the (f) sixth-order polynomial fit.

and comparison of resulting IGWs' analyses from density profiles with those from temperature profiles was discussed with regard to theory.

In the process of deriving our method in Sect. 2.1, we described the quantities defined by Eqs. (3), (4) and (5) as the Brunt-Väisälä or buoyancy frequency. Although this helps to introduce and to understand the separation method, it might be also seen as phenomenologically inconsistent. Such a description would be correct for a fluid where the density is independent of pressure and the density of a fluid particle could be considered unchanged when it was displaced vertically (Sutherland, 2010). In our case this is not true. Therefore, a different naming convention should be used for these quantities in future work. For example, using the density scale height $\left(H_{\rho}\right)$ definition (e.g. Sutherland, 2010), Eq. (3) can be written as

$\frac{g}{H_{\rho}}$.

Nevertheless, the naming convention has no influence on the results.

In Sect. 3.1, the problems of the background choice are discussed, correspondence between analytical forms of density and temperature background profiles is examined and the non-physicality of polynomial temperature fit is shown by solving a related differential equation.
A very important result is shown in Sect. 3.2.1. In the high wave-number region from approximately 0.4 cycle $\mathrm{km}^{-1}$ ( $2.5 \mathrm{~km}$ vertical wavelength), the power spectrum of the normalized fluctuations derived from dry temperature GPS RO data has lower values than for normalized density fluctuations regardless of the background type. Steiner and Kirchengast (2000) argued that the likely causes of a tendency of increasing underestimation of spectral power towards higher wave numbers are the GO technique, the local spherical symmetry assumption and the hydrostatic equilibrium assumption. The last of these is confirmed by our results.

Steiner and Kirchengast (2000) stated that abovementioned causes of underestimation are acting to suppress the wave amplitudes and therefore to lower the PSDs. But this is not the only implication. Filtering the data with the hydrostatic balance in the standard temperature retrieval leads to the fact that in the equations of motions of the filtered field also the dynamic pressure and density fluctuations are in hydrostatic balance. That results in the vanishing of vertical accelerations. The consequence is that the whole group of nonhydrostatic IGWs is filtered out. According to Sutherland (2010), those are waves with frequency close to the buoyancy frequency and waves with phase line slopes significantly different from zero. It should be noted that these waves with smaller ratio of horizontal to vertical wavelength 
may be already filtered by the local spherical symmetry assumption.

In Sect. 3.2.2, the results of CWT and its skeleton do not reveal noticeable differences between the dry temperature and density based IGW analyses. Rather the differences between the scaled and unscaled cases are more important and the role of subjective background choice has visible influence on the longer modes. We do not discuss the propagation or sourcing of waves, which could be tempting especially in the case of modes emerging or ending suddenly in the profile. The GPS RO measurement is a "snapshot" of the real atmosphere and no information from the cotangent space is included. Hence, using just a simple profile, we cannot say if or in which direction the mode propagates. Special care must be taken also when interpreting the results of the CWT skeleton because the vertical behaviour of modes could be also a consequence of fluctuation retrieval rather than of physical processes. That can be seen in Fig. 6 from comparison of the dominant modes between 4 and $8 \mathrm{~km}$ vertical wavelength in the upper stratosphere.

In summary, the analysis of IGWs with the GPS RO density profiles bears advantages over the analysis from dry temperature profile. Primarily, unlike the temperature, the density background has a familiar form and the consequent analysis is not restricted to hydrostatic waves.

Acknowledgements. The study was supported by the Charles University in Prague, project GA UK No. 108313, by the Aktion program, by grant no. SVV-2014-26096, and by the Austrian Science Fund (FWF; BENCHCLIM project P22293-N21. UCAR/CDAAC (Boulder, CO, USA) is acknowledged for the provision of RO data. Finally we thank Andrea K. Steiner (WEGC, University of Graz) for fruitful discussions.

Edited by: J. Y. Liu

\section{References}

Alexander, P., de la Torre, A., Llamedo, P., Hierro, R., Schmidt, T., Haser, A., and Wickert, J.: A method to improve the determination of wave perturbations close to the tropopause by using a digital filter, Atmos. Meas. Tech., 4, 1777-1784, doi:10.5194/amt4-1777-2011, 2011.

Anthes, R. A., Bernhardt, P. A., Chen, Y., Cucurull, L., Dymond, K. F., Ector, D., and Thompson, D. C.: THE COSMIC/FORMOSAT-3 MISSION Early Results, B. Am. Meteorol. Soc., 89, 313-333, doi:10.1175/BAMS-89-3-313, 2008.

Chane-Ming, F., Molinaro, F., Leveau, J., Keckhut, P., and Hauchecorne, A.: Analysis of gravity waves in the tropical middle atmosphere over La Reunion Island $\left(21^{\circ} \mathrm{S}, 55^{\circ} \mathrm{E}\right)$ with lidar using wavelet techniques, Ann. Geophys., 18, 485-498, doi:10.1007/s00585-000-0485-0, 2000.

Chiu, Y. T. and Ching, B. K.: The response of atmospheric and lower ionospheric layer structures to gravity waves, Geophys. Res. Lett., 5, 539-542, 1978.
Cushman-Roisin, B.: Introduction to Geophysical Fluid Dynamics, Prentice Hall, Englewood Cliff, New Jersey 07632, 1994.

Ern, M., Preusse, P., Gille, J. C., Hepplewhite, C. L., Mlynczak, M. G., Russell, J. M., and Riese, M.: Implications for atmospheric dynamics derived from global observations of gravity wave momentum flux in stratosphere and mesosphere, J. Geophys. Res.Atmos., 116, D19107, doi:10.1029/2011JD015821, 2011.

Foelsche, U., Borsche, M., Steiner, A. K., Gobiet, A., Pirscher, B., Kirchengast, G., and Schmidt, T.: Observing upper tropospherelower stratosphere climate with radio occultation data from the CHAMP satellite, Clim. Dynam., 31, 49-65, 2008.

Fritts, D. C. and Alexander, M. J.: Gravity wave dynamics and eff ects in the middle atmosphere, Rev. Geophys., 41, 1003, doi:10.1029/2001RG000106, 2003.

Garcia, R. R. and Randel, W. J.: Acceleration of the Brewer-Dobson circulation due to increases in greenhouse gases, J. Atmos. Sci., 65, 2731-2739, 2008.

Gardner, C. S. and Shelton, J. D.: Density response of neutral atmospheric layers to gravity wave perturbations, J. Geophys. Res.Space, 90, 1745-1754, 1985.

Gardner, C. S., Senft, D. C., Beatty, T. J., Bills, R. E., and Hostetler, C. A.: Rayleigh and sodium lidar techniques for measuring middle atmosphere density, temperature and wind perturbations and their spectra, in: World Ionosphere/Thermosphere Study, Volume 2, edited by: Liu, C. H., Chapter 6, University of Illinois, 1406 W. Green St., Urbana, Illinois 61801. 148-187, December 1989.

Gubenko, V. N., Pavelyev, A. G., and Andreev, V. E.: Determination of the intrinsic frequency and other wave parameters from a single vertical temperature or density profile measurement, J. Geophys. Res.-Atmos., 113, D08109, doi:10.1029/2007JD008920, 2008.

Gubenko, V. N., Pavelyev, A. G., Salimzyanov, R. R., and Pavelyev, A. A.: Reconstruction of internal gravity wave 5 parameters from radio occultation retrievals of vertical temperature profiles in the Earth's atmosphere, Atmos. Meas. Tech., 4, 2153-2162, doi:10.5194/amt-4-2153-2011, 2011.

Gubenko, V. N., Pavelyev, A. G., Salimzyanov, R. R., and Andreev, V. E.: A method for determination of internal gravity wave parameters from a vertical temperature or density profile measurement in the Earth's atmosphere, Cosmic Res., 50, 21-31, doi:10.1134/S0010952512010029, 2012.

Hines, C. O.: Internal atmospheric gravity waves at ionospheric heights, Can. J. Phys., 38, 1441-1481, 1960.

Liou, Y. A., Pavelyev, A. G., Huang, C. Y., Igarashi, K., Hocke, K., and Yan, S. K.: Analytic method for observation of the gravity waves using radio occultation data, Geophys. Res. Lett., 30, 2021, doi:10.1029/2003GL017818, 2003.

Liou, Y. A., Pavelyev, A. G., and Wickert, J.: Observation of the gravity waves from GPS/MET radio occultation data, J. Atmos. Sol.-Terr. Phys., 67, 219-228, 2005.

Luna, D., Alexander, P., and de la Torre, A.: Evaluation of uncertainty in gravity wave potential energy calculations through GPS radio occultation measurements, Adv. Space Res., 52, 879-882, doi:10.1016/j.asr.2013.05.015, 2013.

Markwardt, C. B.: Non-linear least squares fitting in IDL with MPFIT, arXiv preprint arXiv:0902.2850, 2009.

Marquardt, C. and Healy, S. B.: Notes and correspondence: Measurement noise and stratospheric gravity waves characteristics 
obtained from GPS occultation data, J. Meteorol. Soc. Jpn., 83, 417-428, 2005.

Marshall, A. G. and Scaife, A. A.: Impact of the QBO on surface winter climate, J. Geophys. Res.-Atmos., 114, D18110, doi:10.1029/2009JD011737, 2009.

Melbourne, W. G.: Radio occultations using Earth satellites: a wave theory treatment, Vol. 5, Wiley-Blackwell, ISBN 0-471-71222-1, 2005.

Pavelyev, A. G., Wickert, J., Liou, Y. A., Pavelyev, A. A., and Jacobi, J.: Analysis of atmospheric and ionospheric wave structures using the CHAMP and GPS/MET radio occultation database, in: Atmosphere and Climate Studies by Occultation Methods, edited by: Foelsche, U., Kirchengast, G., and Steiner, A., Springer Verlag, Springer Berlin Heidelberg, 225-242, doi:10.1007/3-54034121-8_19, 2006.

Pavelyev, A. G., Liou, Y. A., Wickert, J., Gubenko, V. N., Pavelyev, A. A., and Matyugov, S. S.: New Applications and Advances of the GPS Radio Occultation Technology as Recovered by Analysis of the FORMOSAT-3/COSMIC and CHAMP Data-Base, in: New Horizons in Occultation Research: Studies in Atmosphere and Climate, edited by: Steiner, A., Pirscher, B., Foelsche, U., Kirchengast, G., Springer, Berlin Heidelberg, 165178, doi:10.1007/978-3-642-00321-9, 2009.

Preusse, P., Eckermann, S. D., and Ern, M.: Transparency of the atmosphere to short horizontal wavelength gravity waves, J. Geophys. Res.-Atmos., 113, D24104, doi:10.1029/2007JD009682, 2008.

Schmidt, T., Wickert, J., De la Torre, A., Alexander, P., Faber, A., Llamedo, P., and Heise, S.: The effect of different background separation methods on gravity wave parameters in the upper troposphere and lower stratosphere region derived from GPS radio occultation data, in: 39th COSPAR Scientific Assembly, Vol. 39, July, 1721 pp., 2012.

Schreiner,W., Rocken, C., Sokolovskiy, S., Syndergaard, S., and Hunt, D.: Estimates of the precision of GPS radio occultations from the COSMIC/FORMOSAT-3 mission, Geophys. Res. Lett., 34, L04808, doi:10.1029/2006GL027557, 2007.

Senft, D. C. and Gardner, C. S.: Seasonal variability of gravity wave activity and spectra in the mesopause region at Urbana, J. Geophys. Res.-Atmos., 96, 17229-17264, 1991.

Sica, R. and Russell, A.: Measurements of the eff ects of gravity waves in the middle atmosphere using parametric models of density fluctuations. Part I: vertical wavenumber and temporal, J. Atmos. Sci., 56, 1308-1329, 1999.
Steiner, A. K. and Kirchengast, G.: Gravity Wave Spectra from GPS/MET Occultation Observations, J. Atmos. Ocean. Tech., 17, 495-503, 2000.

Sutherland, B.: Internal Gravity Waves, Cambridge University Press, ISBN:9780521839150, 2010.

Tsuda, T. and Hocke, K.: Vertical wave number spectrum of temperature fluctuations in the stratosphere using GPS occultation data, J. Meteorol. Soc. Jpn., 80, 925-938, doi:10.2151/jmsj.80.925, 2002.

Tsuda, T., Nishida, M., Rocken, C., and Ware, R. H.: A global morphology of gravity wave activity in the stratosphere revealed by the GPS occultation data (GPS/MET), J. Geophys. Res.-Atmos., 105, 7257-7273, 2000.

Tsuda, T., Lin, X., Hayashi, H., and Noersomadi: Analysis of vertical wave number spectrum of atmospheric gravity waves in the stratosphere using COSMIC GPS radio occultation data, Atmos. Meas. Tech., 4, 1627-1636, doi:10.5194/amt-4-1627-2011, 2011.

Torrence, C. and Compo, G. P.: A practical guide to wavelet analysis, B. Am. Meteorol. Soc., 79, 61-78, 1998.

Vincent, R. A., Allen, S. J., and Eckermann, S. D.: Gravity-wave parameters in the lower stratosphere, in: Gravity Wave Processes, Springer, Berlin Heidelberg, 7-25, 1997.

Wang, L. and Alexander, M. J.: Global estimates of gravity wave parameters from GPS radio occultation temperature data, J. Geophys. Res.-Atmos., 115, D21122, doi:10.1029/2010JD013860, 2010.

Wickert J., Schmidt T., Michalak G., Heise S., Arras C., Beyerle G., Falck C., König R., Pingel D., and Rothacher M.:GPS radio occultation with CHAMP, GRACE-A, SAC-C, TerraSAR-X, and FORMOSAT-3/COSMIC: brief review of results from GFZ, in: New horizons in occultation research, edited by: Steiner, A., Pirscher, B., Foelsche, U., Kirchengast, G., Springer, Berlin Heidelberg, 3-15, doi:10.1007/978-3-642-00321-9, 2009.

Wilson, R., Chanin, M. L., and Hauchecorne, A.: Gravity waves in the middle atmosphere observed by Rayleigh lidars. Part 2: Climatology, J. Geophys. Res., 96, 5169-5183, 1991.

Wu, D. L., Preusse, P., Eckermann, S. D., Jiang, J. H., Juarez, M. D. L. T., Coy, L., and Wang, D. Y.: Remote sounding of atmospheric gravity waves with satellite limb and nadir techniques, Adv. Space Res., 37, 2269-2277, 2006. 